\section{Changes in the incidence and epidemiology of neonatal group B Streptococcal disease over the last two decades in Crete, Greece}

\author{
Eleni Vergadi, ${ }^{1}$ Antonia Manoura, ${ }^{2}$ \\ Emmanouil Chatzakis, ${ }^{3}$ \\ Emmanouil Karavitakis, ${ }^{4}$ \\ Sofia Maraki, ${ }^{5}$ Emmanouil Galanakis ${ }^{1}$
}

${ }^{1}$ Department or Pediatrics, University of Crete, School of Medicine; ${ }^{2}$ Department of Neonatology, Heraklion University

Hospital, ${ }^{3}$ Department of Neonatology,

Venizeleion General Hospital;

${ }^{4}$ Department of Neonatology, General

Hospital of Chania; ${ }^{5}$ Department of

Clinical Microbiology, Heraklion

University Hospital, Crete, Greece

\begin{abstract}
Group B streptococcus (GBS) remains a leading cause of neonatal disease. However, GBS rates and prevention strategies vary considerably worldwide. Herein, we investigated the burden and epidemiological trends of neonatal GBS infections in our area (Greece) over the last two decades. We conducted a multicenter retrospective study that includes all cases of culture-proven GBS disease in infants $<90$ days old in the last 22 years. Neonatal GBS incidence was $0.17 / 1000$ live births (95\%CI: $0.11-0.21)$. A significant increase was noted during the second decade $(0.23$ vs $0.10 / 1000, \mathrm{P}<0.05)$. Late onset disease (LOD) significantly increased during the second decade $(0.08 v \mathrm{~s}$ $0.02, \mathrm{P}<0.05)$. Infants in the LOD group had a higher risk of meningitis (RR 1.8, 95\%CI: 1.23-2.71). Long-term neurological sequelae were reported in $42.8 \%$ of meningitis cases. The mortality rate was $8 \%$. The incidence of neonatal GBS disease in our area is among the lowest reported, but an increase was noted the last decade mainly due a rise in the LOD. The burden of LOD, the mortality and long-term disability are still substantial, thus effective prevention strategies - including maternal vaccination for neonatal GBS - are needed.
\end{abstract}

\section{Introduction}

Group B Streptococcus (GBS), or Streptococcus agalactiae, is a leading cause of sepsis and meningitis in neonates and young infants, with substantial mortality and morbidity including long term neurode- velopmental sequelae. ${ }^{1}$ Invasive neonatal GBS infection can be classified as earlyonset disease (EOD) when it occurs within the first seven days of life, and late onset disease (LOD) when it develops after the first week of life until 90 days old. $^{2}$

Neonates born from GBS-colonized mothers often become colonized during or before delivery and may develop invasive disease. Following the guidelines for prevention of perinatal GBS disease with universal antenatal screening and/or intrapartum antibiotic prophylaxis for culturepositive and high-risk pregnant women, the incidence of neonatal EOD has significantly declined. ${ }^{3,4}$ However, there is a substantial variability of GBS incidence rates worldwide; in several countries GBS disease affects a significant number of infants, while in others GBS burden remains low. ${ }^{5-8}$

Research has recently focused on additional strategies for GBS prevention, such as the development of a vaccine. Knowledge of local epidemiology is required to assess the role of prevention and the potential impact of GBS vaccination in pregnant women. Since information on the incidence of GBS neonatal disease is scarce in our country, we assessed GBS epidemiology in neonates and young infants less than 90 days of age in the island of Crete, the largest island of Greece, and we estimated the burden of GBS disease in terms of incidence, mortality and long-term morbidity over a 22-year period.

\section{Materials and Methods}

Study period, study participants, and definitions

A case was defined by the isolation of GBS from a normally sterile site on day 089 of life in an infant born in Crete. All culture-proven cases of GBS invasive disease were retrospectively included. These infants were managed in all the hospitals that have neonatal intensive care units and pediatric departments, in the study area during a 22year period, from January 1995 to December 2016. The included hospitals do not only offer intrapartum services, but are also referral centers for pregnant or parturient women and neonates that have been followed either at public or private nursery centers. Microbiological records were reviewed to confirm the diagnosis.

Clinical variables included gender, age at presentation, birth weight, gestational age, delivery mode, time of membrane rupture, previous maternal hospitalization and number of deaths. Medical records of infants with GBS meningitis were addition-
Correspondence: Emmanouil Galanakis, Department of Pediatrics, Heraklion University Hospital, Crete, 71500 Greece.

Tel.: +30.2810392012; Fax: +30.2810392827. E-mail: emmgalan@uoc.gr

Key words: neonatal infections, Group B streptococcus, incidence, late onset, early onset, intrapartum prophylaxis.

Contributions: EV, AM, EC, EK and SM collected the case information, EG was involved in planning, drafted the manuscript and supervised the work, EV processed the experimental data, interpreted the results, performed the analysis, drafted the manuscript and designed the figures. All authors discussed the results and commented on the manuscript and contributed to the writing of the manuscript.

Conflict of interest: the authors declare no conflict of interest.

Funding: none.

Received for publication: 12 May 2018.

Revision received: 5 July 2018.

Accepted for publication: 28 June 2018 .

This work is licensed under a Creative Commons Attribution-NonCommercial 4.0 International License (CC BY-NC 4.0).

CCopyright E. Vergadi et al., 2018

Licensee PAGEPress, Italy

Infectious Disease Reports 2018; 10:7744

doi:10.4081/idr.2018.7744

ally reviewed to assess long-term outcomes. Furthermore, we performed consented parental interviews using a standardized questionnaire that assessed the developmental outcome of GBS subjects with meningitis, including the presence of seizures, movement disabilities, speech delay and learning disabilities, cognitive or behavioral problems and vision or hearing screening. This study was conducted according to the principles of the Declaration of Helsinki and in accordance with the Medical Research Involving Human Subjects Act (WMO).

\section{Statistical analysis}

The information was used to calculate annual incidence rates (rate per 1000 live births) for EOD and LOD, using data on the total number of live births in Crete as provided by the national statistical authority. ${ }^{9}$ All data were evaluated for normality using Kolmogorov - Smirnov test (with Dallal Wilkinson - Lilliefor P value). The samples that passed the normality test were analyzed with parametric tests and the rest with non- 
parametric ones. The comparison of results between different groups was performed by t-test or one-way analysis of variance with Bonferroni's multiple comparison post test for normally distributed data and Mann Whitney U test or Kruskal-Wallis test with Dunn's multiple comparison post test for non-parametric data. Analysis of categorical data was performed with Fisher's exact test. P-value $<0.05$ was considered significant. Analysis was made using the GraphPad InStat software (GraphPad, San Diego, CA).

\section{Results}

During the 22-year period, there were 148,124 live births in Crete, and 25 neonates and young infants were affected by an invasive GBS infection. The overall incidence of GBS disease was $0.17 / 1000$ live births ( $95 \%$ CI 0.11 to 0.21$)$ and varied from 0.0 to 0.374 annually (Figure 1). The overall incidence of EOD and LOD was 0.11 (95\%CI 0.06 to 0.21$)$ and $0.06(95 \% \mathrm{CI}$ 0.25 to 0.10 ) respectively (Figure 1 ). There were no recurrent GBS cases during the study period.

In pre-term newborns, before 37 weeks of gestational age, GBS incidence was 0.37 per 1000 live births $(95 \% \mathrm{Cl} 0.06$ to 0.68$)$ and was significantly higher compared to overall GBS incidence $(\mathrm{P}=0.025)$. In infants born at term, after 37 weeks of gestational age, GBS incidence was 0.12 (95\%CI 0.07 - 0.17) per 1000 live births.

Interestingly, a significant increase in GBS incidence was noted during the last 11 years compared to the previous decade. In the period from 2006 to 2016, GBS rates raised to $0.23 / 1000$ live births compared to $0.10 / 1000$ live births during the period $1995-2005, \mathrm{P}<0.05)$. Most cases were of EOD (incidence 0.10/1000) while LOD (incidence $0.06 / 1000$ ) were significantly increased during the last decade (0.08 vs $0.02, \mathrm{P}<0.05$, Figure 2).

The infants' clinical characteristics are depicted in Table 1. The median age of presentation of GBS infection was 2 days (range 0-77), median birth weight was $3,150 \mathrm{~g}$ (range 1100-4250) and median gestational age 38.8 weeks (range 24.5-40). Among infants with GBS infection, $7 / 25$ were born with cesarean section, $8 / 25$ presented with meningitis (median age 5 days, range 1-21) and the remaining $17 / 25$ with septicemia. Most cases were of EOD $(16 / 25,64 \%)$ presenting at the ages of $0-3$ (median 1) days and commonly within 24 hours of birth. The remaining nine cases were of LOD and presented between days 7 and $28(6 / 25,24 \%)$ and between days $28-90(3 / 25,12 \%)$.

Among the subjects with EOD, five $(5 / 16,31.25 \%)$ had risk factors for infection. Risk factors that were noted were prematurity (2/16), prolonged rupture of membranes above 18 hours $(2 / 16)$, or both $(1 / 16)$ (Table 1). Among the nine cases of LOD at the age of 7-77 (median 18) days, four $(44.4 \%)$ had a history of previous hospitalization (Table 1). One death was reported in the EOD group and one death in the LOD group. The overall case fatality rate was $8 \%$ (2/25).

In the LOD group, meningitis cases were more common compared to EOD group (44.4\% vs 25\%, $\mathrm{P}=0.007$ ) (Figure 3). Infants with LOD had a higher risk of presenting with meningitis compared to infants with EOD (RR 1.8, 95\%CI: 1.23-2.71).

Meningitis was diagnosed in $32 \%$ $(8 / 25)$ of cases (males 6/8, 75\%) in median age of 5 days (range 1-21) and half of them $(4 / 8,50 \%)$ presented with seizures. Infants with GBS meningitis were further assessed for long-term developmental sequelae. Outcome information was available in seven out of the eight meningitis cases. The median age at evaluation was 8.6 years (range 4.1-17.3 years). Four children were normal at evaluation, while three of them $(42.8 \%)$ were found to have developmental impairments. Specifically, one infant developed cerebral palsy (quadriplegia) and mental retardation, one obstructive hydrocephalus with minor disability (hemiparesis) and another one speech delay without cognitive defects.

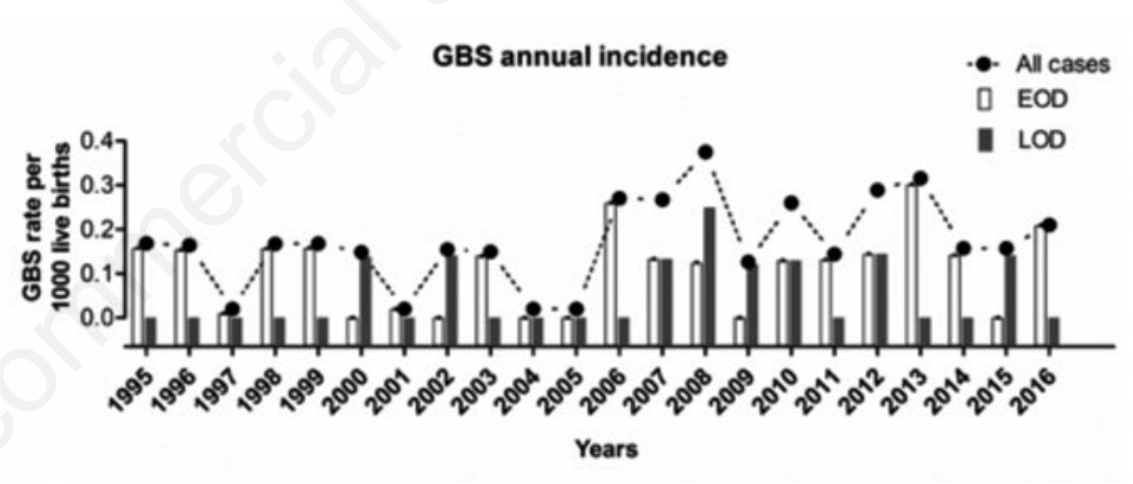

Figure 1. Annual incidence of Group B Streptococcus (GBS) infections in infants less than 90 days of age in Crete, Greece. The rate of all cases of GBS are depicted (circles, black line) as well as the incidence of early onset GBS disease (EOD, white bars) and late onset GBS disease (LOD, gray bars) per each year.

Table 1. Characteristics of infants less than 90 days old with Group B Streptococcus (GBS) invasive disease.

\begin{tabular}{|c|c|c|c|c|}
\hline & Total & Early Onset & Late Onset & p values \\
\hline GBS invasive disease & & $16 / 25(64 \%)$ & $9 / 25(36 \%)$ & \\
\hline Male sex & $13 / 25(52 \%)$ & $8 / 16(50 \%)$ & $5 / 9(55,5 \%)$ & ns \\
\hline Gestational age (weeks, median) & 38.8 & 38.1 & 38.8 & ns \\
\hline Prematurity (\%) & $5 / 25(20 \%)$ & $3 / 16(18,7 \%)$ & $2 / 9(22,2 \%)$ & ns \\
\hline Birth weight $<1500 \mathrm{gr}$ & $5 / 25(20 \%)$ & $3 / 16(18,7 \%)$ & $2 / 9(22,2 \%)$ & ns \\
\hline Cesarean section (\%) & $7 / 25(28 \%)$ & $3 / 16(18,7 \%)$ & $4 / 9(44,4 \%)$ & $<0.001$ \\
\hline Age at diagnosis (days, median) & $2(0-77)$ & $1(0-3)$ & $18(7-77)$ & $<0.001$ \\
\hline Premature rupture of membranes & $5 / 25(20 \%)$ & $3 / 16(18,7 \%)$ & $2 / 9(22,2 \%)$ & ns \\
\hline Meningitis & $8 / 25(32 \%)$ & $4 / 16(25 \%)$ & $4 / 9(44,4 \%)$ & 0.007 \\
\hline Deaths & $2 / 25(8 \%)$ & $1 / 16(6,2 \%)$ & $1 / 9(11,1 \%)$ & ns \\
\hline
\end{tabular}




\section{Discussion}

In this study, we describe the incidence, long-term morbidity and mortality rates of GBS invasive disease in infants under 90 days of age in Crete. Over a 22-year period, a low overall incidence of GBS disease of 0.17 per 1000 live births was observed. Incidence was higher in premature infants, but still low compared to previous reports. ${ }^{10,11}$ However, over the last decade, a 4-fold rise in LOD was noted that resulted in a 2.3-fold increase in the total GBS incidence in this age group.

The GBS incidence presented in this study is among the lowest reported so far; the mean GBS incidence worldwide has been estimated at 0.53 per 1000 live births (95\% CI 0.44-0.62), ${ }^{8}$ ranging approximately from 0.15 to $3.00 .^{5-8,12-14}$ Information on GBS incidence in Greece is scarce up to now. Limited evidence based on physician records and short periods of observation has shown rates from 0.15 to 0.26 per 1000 live births,${ }^{10}$ in accordance with our findings.

The variations in GBS incidence have been attributed to several factors, such as differences in maternal GBS carriage and GBS antibody levels, lifestyle, genetic susceptibility, GBS strain virulence or delivery outside hospitals that may lead to unrecognized causes. ${ }^{15,16}$ Studies on GBS carriage in Greek women have revealed rates between $4 \%$ and $12 \%,{ }^{9,17-19}$ which are lower compared to the rates reported in other countries (18 to $37 \%$ ) with a higher GBS incidence. ${ }^{20-23}$ Low rates of transmission from mothers to newborns $(19-22 \%)$ have also been observed in Greece. ${ }^{10}$ However, given the retrospective design of our study, a few cases may have not been recorded.
Furthermore, we have no data for culturenegative cases or stillbirths due to GBS.

A substantial increase in perinatal GBS rates was observed during the last years of the study. Preventive measures and recommendations had a significant impact on the rates of GBS in many countries. ${ }^{15,16}$ Guidelines for GBS prevention have been issued by CDC in 1996, followed by national guidelines in many countries worldwide that led to up to a $70 \%$ decrease in the incidence of EOD. ${ }^{1}$ However, in contrast with other countries where prevention strategies have been implemented, we did not observe a decrease of EOD in our area. In Greece, no national policy on GBS prevention measures has been issued, although the universal culture-based screening program and the intrapartum antibiotic prophylaxis are generally suggested. Failure of a risk-based approach of intrapartum chemoprophylaxis and a rise in rates of GBS invasive disease have been also recently reported in UK. ${ }^{24}$

Importantly, we noticed a significant increase in GBS LOD invasive infections, that in generally are not affected by prenatal and intrapartum prevention strategies. ${ }^{25}$ Several risk factors have been associated with LOD. Specifically, LOD due to GBS was found to be associated with hospitalization in infants and indeed almost half of the infants with LOD in our study $(44.4 \%, 4$ out of 9) had a history of previous hospitalization. ${ }^{26}$ Additionally, several case reports have proposed that GBS-infected breast milk may also be a risk factor for LOD and may actually be a cause of LOD even if perinatal preventive measures have been applied. ${ }^{26-28}$ However, we have no information on the breastfeeding practices and on GBS cultures in the maternal milk in the study subjects. Thus, we cannot assess the potential role of breastfeeding and milk contamination on the increase of LOD.

In infants with GBS meningitis, neurological sequelae were considerable, as neurological disability was observed in $42.5 \%$ of infants. This proportion is also comparable to that of recently published studies on long-term evaluation of infants who survived from GBS meningitis..$^{1,29,30}$ The casefatality rate for GBS neonatal disease is estimated at 1.4 to $18 \%$ in recent reports, $, 5,7,8,12$ and was $8 \%$ in our study.

Our results reveal a very low incidence of GBS EOD and LOD in neonates and infants less than 90 days old, lower than the rates reported by many other studies worldwide. However, even if the incidence of GBS neonatal disease remains low in our area, the burden of GBS LOD remains substantial and increases the risk of GBS meningitis. The burden of GBS disease is also reflected in considerably high longterm disability rates

\section{Conclusions}

The above results raise important questions on the value of the existing preventing measures in countries with a low GBS incidence. Since LOD cannot be avoided by prenatal and intrapartum preventive measurements, in the areas with low incidence but substantial burden of LOD, the application of maternal GBS vaccination may be still necessary and important. Further research is needed in our region to determine the potential benefit of new preventive approaches, such as GBS maternal vaccination, on the effective elimination of not only EOD but also LOD burden.

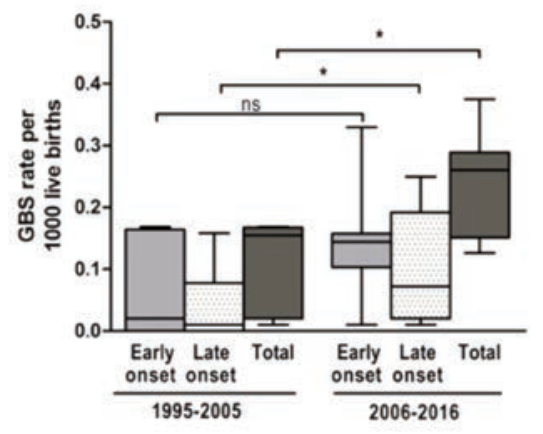

Figure 2. Group B Streptococcus (GBS) rates of early onset disease (EOD), late onset disease (LOD) and total rates of GBS invasive disease in infants less than 90 years old between the decades 1995-2005 and 2006-2016 in Crete, Greece. Boxes show 5-95 percentiles, horizontal line represents median, and whiskers represent minimum and maximum. ${ }^{*} \mathrm{P}<0.05$.

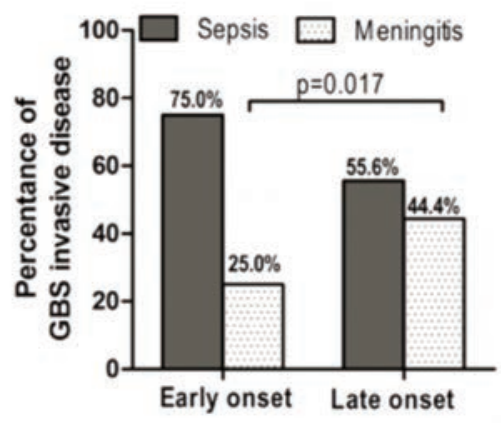

Figure 3. Percentage of Group B Streptococcus (GBS) invasive disease (sepsis and meningitis) in infants born to Crete the last 22 years. Data are categorized to early onset (0-6 days) or late onset (7-89 days) infections. 


\section{References}

1. Libster R, Edwards KM, Levent F, et al. Long-term outcomes of group B streptococcal meningitis. Pediatrics 2012; 130:e8-15.

2. Verani JR, McGee L, Schrag SJ. Prevention of perinatal group B streptococcal disease--revised guidelines from CDC, 2010. MMWR Recomm Rep 2010;59:1-36.

3. Schrag SJ, Whitney CG, Schuchat A. Neonatal group B streptococcal disease: how infection control teams can contribute to prevention efforts. Infect Control Hosp Epidemiol 2000;21:47383.

4. Ecker KL, Donohue PK, Kim KS, et al. The impact of group B Streptococcus prophylaxis on early onset neonatal infections. J Neonatal Perinatal Med 2013;6:37-44.

5. Matsubara K, Hoshina K, Kondo M, et al. Group B streptococcal disease in infants in the first year of life: a nationwide surveillance study in Japan, 20112015. Infection 2017;45:449-58.

6. Sigauque B, Kobayashi M, Vubil D, et al. Invasive bacterial disease trends and characterization of group B streptococcal isolates among young infants in southern Mozambique, 2001-2015. PLoS One 2018;13:e0191193.

7. Guan X, Mu X, Ji W, et al. Epidemiology of invasive group B streptococcal disease in infants from urban area of South China, 2011-2014. BMC Infect Dis 2018;18:14.

8. Edmond KM, Kortsalioudaki C, Scott $\mathrm{S}$, et al. Group B streptococcal disease in infants aged younger than 3 months: systematic review and meta-analysis. Lancet 2012;379:547-56.

9. Hellenic Statistical Authority. Live births in Greece, by permanent residence of mother. Available from: http://www.statistics.gr/en/statistics//publication/SPO03.

10. Tsolia M, Psoma M, Gavrili S, et al. Group B streptococcus colonization of Greek pregnant women and neonates: prevalence, risk factors and serotypes. Clin Microbiol Infect 2003;9:832-8.

11. Risso FM, Castagnola E, Bandettini R, et al. Group B Streptococcus late onset sepsis in very low birth weight newborns: 10 years experience. J Matern Fetal Neon Med 2016:1-10.

12. Giannoni E, Berger C, Stocker M, et al. Incidence and Outcome of Group B Streptococcal Sepsis in Infants in Switzerland. Pediatr Infect Dis J 2016;35:222-4.

13. Dagnew AF, Cunnington MC, Dube Q, et al. Variation in reported neonatal group B streptococcal disease incidence in developing countries. Clin Infect Dis.2012;55:91-102.

14. Melin P, Efstratiou A. Group B streptococcal epidemiology and vaccine needs in developed countries. Vaccine. 2013;31:D31-42.

15. Schuchat A. Group B streptococcus. Lancet 1999;353:51-6.

16. Johri AK, Paoletti LC, Glaser P, et al. Group B Streptococcus: global incidence and vaccine development. Nat Rev Microbiol 2006;4:932-42.

17. Maniatis AN, Palermos J, Kantzanou $\mathrm{M}$, et al. Streptococcus agalactiae: a vaginal pathogen? J Med Microbiol 1996;44:199-202.

18. Daskalakis G, Papapanagiotou A, Mesogitis S, et al. Bacterial vaginosis and group B streptococcal colonization and preterm delivery in a low-risk population. Fetal Diagn Ther 2006;21:1726.

19. Iavazzo C, Vogiatzi C, Falagas ME. A retrospective analysis of isolates from patients with vaginitis in a private Greek obstetric/gynecological hospital (2003-2006). Med Sci Monit 2008;14:CR228-31.

20. Sinha A, Russell LB, Tomczyk S, et al. Disease Burden of Group B Streptococcus Among Infants in SubSaharan Africa: A Systematic Literature Review and Meta-analysis. Pediatr Infect Dis J 2016;35:933-42.

21. Campbell JR, Hillier SL, Krohn MA, et al. Group B streptococcal colonization and serotype-specific immunity in pregnant women at delivery. Obstet Gynecol 2000;96:498-503.

22. Baker CJ. The spectrum of perinatal group B streptococcal disease. Vaccine 2013;31:D3-6.

23. Kwatra G, Cunnington MC, Merrall E, et al. Prevalence of maternal colonisation with group B streptococcus: a systematic review and meta-analysis. Lancet Infect Dis 2016;16:1076-84.

24. Lamagni TL, Keshishian C, Efstratiou $\mathrm{A}$, et al. Emerging trends in the epidemiology of invasive group B streptococcal disease in England and Wales, 1991-2010. Clin Infect Dis 2013;57:682-8.

25. Tzialla C, Borghesi A, Longo S, Stronati M. Which is the optimal algorithm for the prevention of neonatal early-onset group B streptococcus sepsis? Early Hum Dev 2014;90:S35-8.

26. Berardi A, Rossi C, Lugli L, et al. Group B streptococcus late-onset disease: 2003-2010. Pediatrics 2013;131: e361-8.

27. Berardi A, Guidotti I, Creti R, et al. Two Overlapping Clusters of Group B Streptococcus Late-Onset Disease in a NICU. Pediatr Infect Dis J 2018 [Epub ahead of print].

28. Zimmermann P, Gwee A, Curtis N. The controversial role of breast milk in GBS late-onset disease. J Infect 2017;74: S34-S40.

29. Levent F, Baker CJ, Rench MA, Edwards MS. Early outcomes of group B streptococcal meningitis in the $21 \mathrm{st}$ century. Pediatr Infect Dis J 2010;29: 1009-12.

30. Bedford H, de Louvois J, Halket S, et al. Meningitis in infancy in England and Wales: follow up at age 5 years. BMJ 2001;323:533-6. 